\title{
Erratum
}

\section{The expression of Usp26 gene in mouse testis and brain}

\author{
Jie Zhang ${ }^{1}$, Hong Tian ${ }^{1,2}$, Yong-Wei Huo ${ }^{1,2}$, Dang-Xia Zhou ${ }^{3}$, Hai-Xu Wang ${ }^{1}$, Li-Rong Wang ${ }^{1,2}$, Qiu-Yang Zhang ${ }^{1,2}$, \\ Shu-Dong Qiu ${ }^{1,2}$
}

Asian Journal of Andrology (2009) 11: 748-749. doi: 10.1038/aja.2009.73.

Correction to: Asian Journal of Andrology (2009) 11: 478-483. doi: 10.1038/aja.2009.31; published online 8 June 2009

In this article, published on 1 July 2009, the following colored figures was wrongly published in black and white.

Asian Journal of Andrology would like to apologize to the authors and readers for the error and any confusion this may have caused.
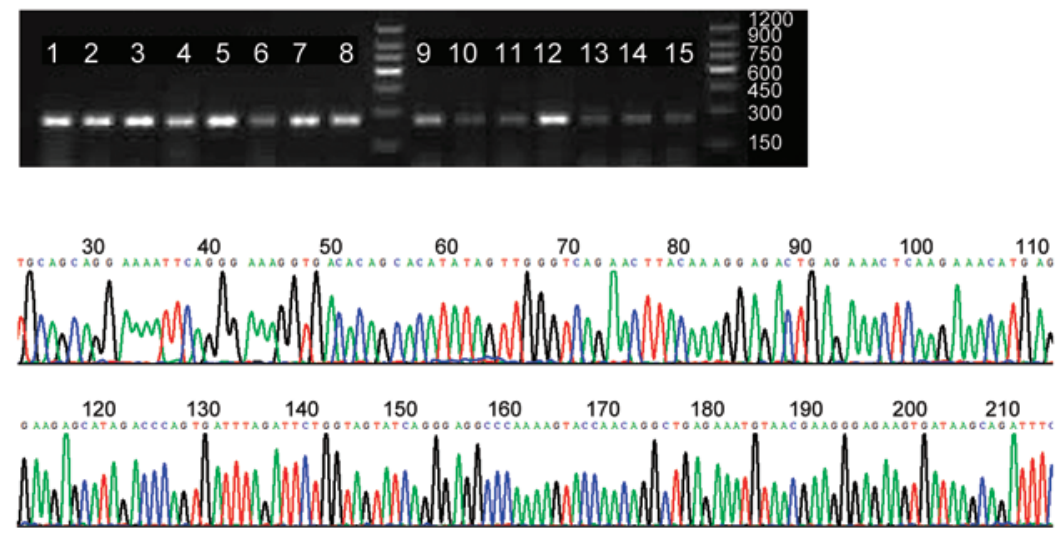

Figure 1. (A) Reserve-transcription polymerase chain reaction (RT-PCR) in multiple mouse tissues. 1, 3, 5 and 7: testes of postnatal days 20,30, 35 and 45, respectively; 2, 4, 6 and 8: epididymides of postnatal days 20,30, 35 and 45, respectively; 9-15: mouse brain, lung, heart, adrenal gland, liver, kidney and seminal gland, respectively, on postnatal day 35. (B) Sequencing results showed that these sequences agreed very well (99\%) with Usp26 mRNA of mouse.
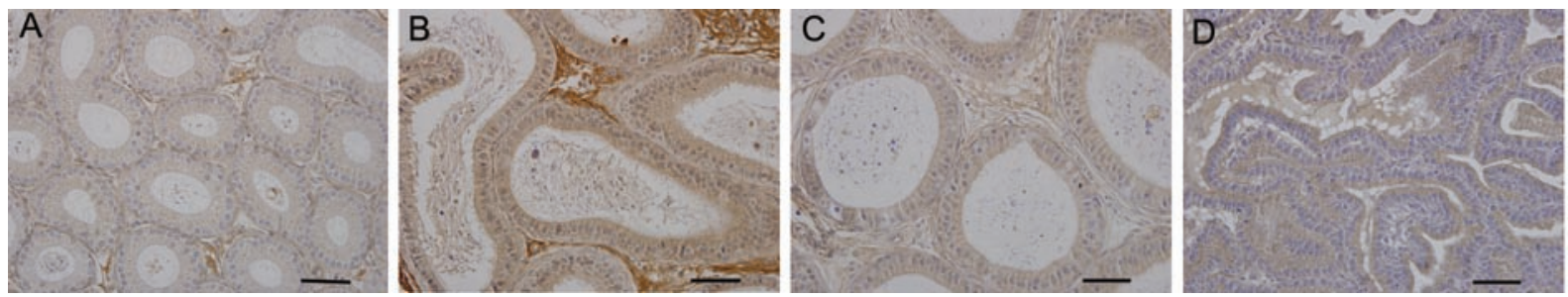

Figure 3. Immunohistochemical localization of USP26 in epididymis (A-C) and seminal gland (D) of a 35-day-old mouse. USP26 is expressed in the epithelium of the two tissues. The staining is stronger in the corpus region (B) than in the caput and cauda regions of epididymis (A and C). Scale bars $=50 \mu \mathrm{m}$. 

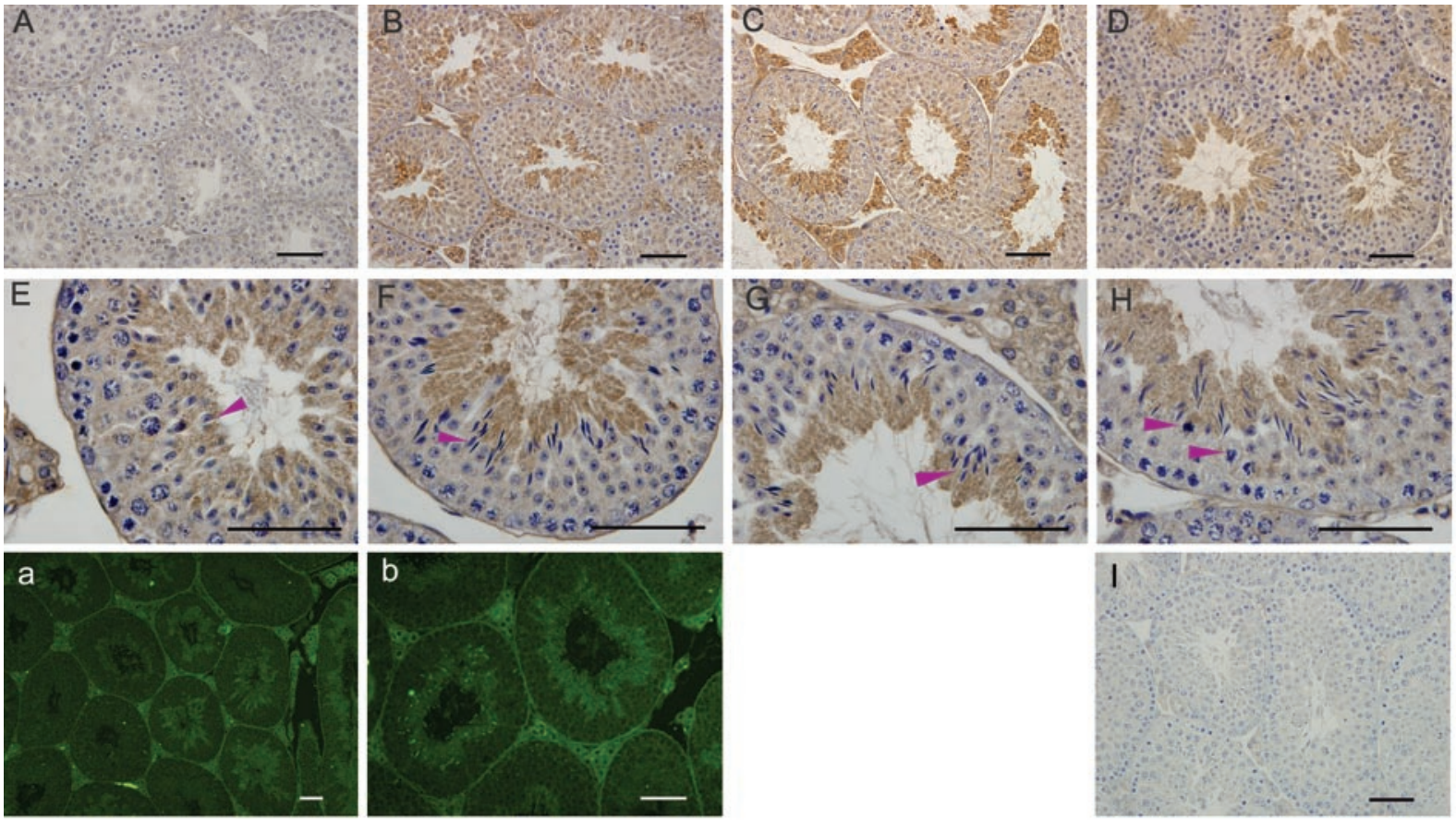

Figure 2. Immunolocalization of ubiquitin-specific protease 26 (USP26) protein in the developing mouse testis. USP26-positive cells are characterized by brown staining as a result of the DAB colorimetric reaction in (A-H) and in green as a result of FITC immunofluorescence staining in (a) and (b). USP26 immunostaining is absent in 20 day (A), weak in 30 day (B) and intense in 35 and 45-day mouse testes, in which the staining is strong in the cytoplasm of condensing spermatids (steps 9-16) (C and D). (E-G) show seminiferous epithelium at late $(\mathrm{E})$ and early stages $(\mathrm{F}, \mathrm{G})$ respectively, and the arrowheads in $(\mathrm{H})$ point to cells undergoing meiotic metaphase. Immunofluorescence staining in panels (a) and (b) also shows USP26 expression in spermatids and in Leydig cells in testes. I: Control section. Scale bars $=50 \mu \mathrm{m}$.
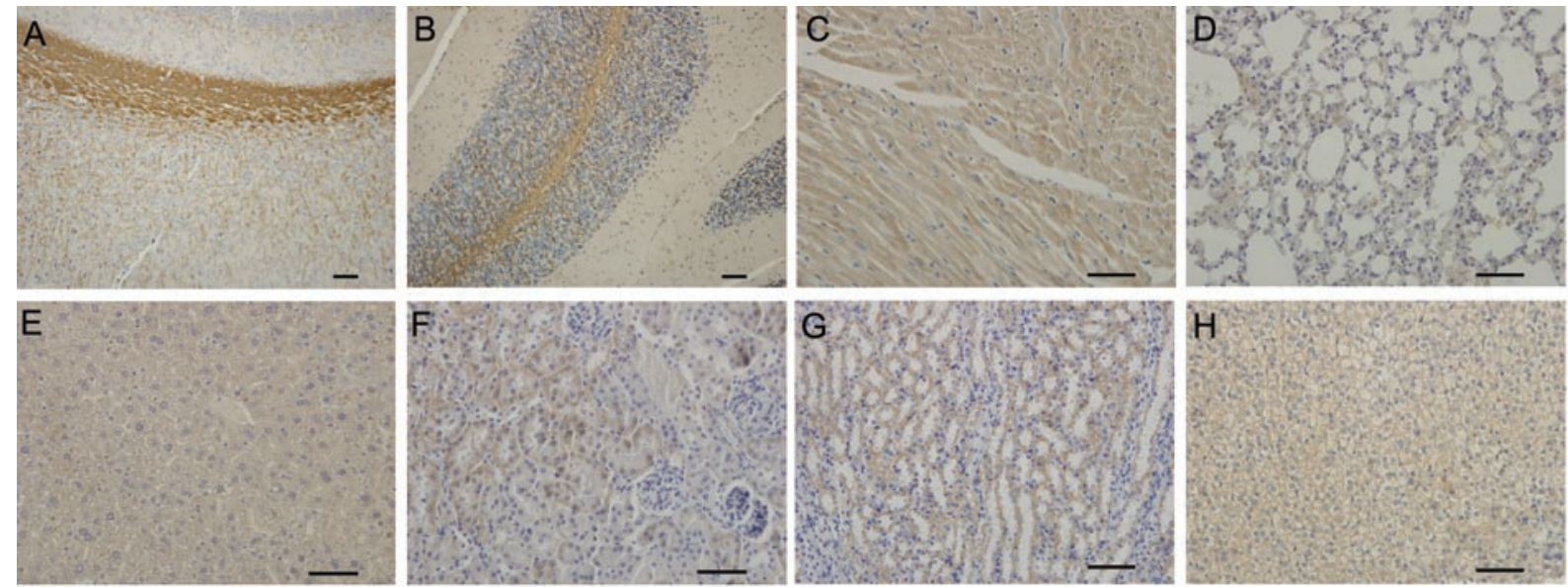

Figure 4. Immunohistochemical staining of USP26 in brain, heart, lung, liver, kidney and adrenal gland of 35-day-old mice. Nerve fibers of the cerebra and cerebellum (A and B) show intense immunoreactive staining, especially in cingulum and external capsule of the cerebra and medulla of the cerebellum. USP26-positive staining is detected at a moderate level in myocardial cells and the epithelium of renal tubule $(\mathrm{C}, \mathrm{F}, \mathrm{G})$, and at a lower level in lung, liver and adrenal gland, respectively $(\mathrm{D}, \mathrm{E}, \mathrm{H}) . \mathrm{Scale}$ bars $=50 \mu \mathrm{m}$. 